\title{
Artículo de revisión \\ Semiología y diagnóstico diferencial de la insuficiencia cardíaca crónica
}

\author{
Héctor Jairo Umaña-Giraldo ${ }^{1} J_{\text {iménez-Salazar Silvana }}^{2}$. sijimenez@utp.co Buitrago-Toro Kenny, ${ }^{2}$ Echeverry-Bolaños Melissa ${ }^{3}$
}

1 Departamento de Medicina Interna HUSJ. Facultad de Salud. Universidad Tecnológica de Pereira.

2 Residente de medicina interna. Universidad Surcolombiana. Hospital universitario Hernando Moncaleano Perdomo.

3 Departamento de Cirugía HUSJ

Fecha de envío: 22/11/2016

Fecha de correcciones 10/01/2017

Fecha de aceptación 27/03/2017

Fecha de publicación: 31/01/2018

Resumen: la insuficiencia cardiaca es un síndrome clínico de etiología multifactorial de alta prevalencia en la población mundial y latinoamericana debido al aumento en la expectativa de vida. Actualmente el diagnóstico de este se basa en los hallazgos de pruebas diagnósticas como el ecocardiograma o pépticos natriuréticos, pero es importante para el personal médico conocer e identificar de manera clara los signos y síntomas que presentan los pacientes con insuficiencia cardiaca, pues es una tendencia actual el desconocer datos semiológicos importantes que fueron descritos y refinados durante los siglos XIX y XX, y que ahora son validados por la medicina basada en la evidencia. Lo anterior cobra importancia pues el papel que debe tener la creciente tecnología no es acabar o desplazar el conocimiento clínico, si no ser una herramienta complementaria para el buen cuidado del paciente y así llegar al mejor desenlace posible.

Este artículo pretende brindar datos basados en la evidencia para hacer de la clínica la parte fundamental del proceso diagnóstico y de la relación médico paciente. También intenta ser una guía útil para estudiantes de medicina o médicos que se desenvuelven en sitios con pocos recursos económicos, ya que la elección adecuada de un examen diagnóstico solo puede ser llevada a cabo mediante un buen interrogatorio y examen físico.

Palabras clave: insuficiencia cardiaca; Diagnóstico; Signos y Síntomas; Colombia

Copyright ( $\odot$ Facultad de Ciencias de la Salud de la Universidad Tecnológica de Pereira. 1995-2017. Todos los derechos reservados ${ }^{*}$

\section{Semiology and differential diagnosis of chronic heart failure}

Abstract: heart failure is a clinical syndrome with multifactorial etiology of high prevalence in the world and Latin American population due to the increase in life expectancy. Currently the diagnosis of this is based on the findings of diagnostic tests such as echocardiogram or natriuretic peptic, but it is important for the medical staff to know and clearly identify the signs and symptoms that patients with heart failure present, as it is a current trend To ignore important semiological data that were described and refined during the nineteenth and twentieth centuries, and which are now validated by evidencebased medicine. This is important because the role of growing technology is not to end or displace clinical knowledge, if not to be a complementary tool for good patient care and thus reach the best possible outcome.

This article aims to provide data based on the evidence to make the clinic the fundamental part of the diagnostic process and the patient medical relationship. It is also intended to be a useful guide for medical students or physicians operating in poorly funded areas, as the proper choice of a diagnostic examination can only be carried out through a thorough examination and physical examination.

Key words: heart failure; Diagnosis; Signs and Symptoms; Colombia

Copyright $\odot$ Facultad de Ciencias de la Salud de la Universidad Tecnológica de Pereira. 1995-2017. All rights reserved ${ }^{\circ}$.

\section{Introducción}

La insuficiencia cardiaca (IC) es un importante problema de salud pública a nivel mundial, con una prevalencia estimada de 23 millones de personas afectadas[1,2]. El riesgo de desarrollar IC a lo largo de la vida es del 20\% para las personas mayores de 40 años[2]. La incidencia aumenta con la edad, de 20 casos por 1000 habitantes para las personas de 65-69 años a más de 80 casos por 1000 habitantes para las personas mayores de 85 años[3]. Se presenta más en la raza negra seguido por hispanos y norteamericanos[1].

En Latinoamérica la incidencia de insuficiencia cardiaca va en aumento, debido a una mayor expectativa de vida, zonas endémicas para enfermedad de Chagas[4], avances en el tratamiento de enfermedades cardiovasculares y a la pandemia de síndrome metabólico que vive actualmente la población hispana[5].

La IC es un síndrome clínico progresivo que puede tener diferentes características dependiendo de la etiología, edad, sexo, raza, etnia, y fracción de eyección del ventrículo izquierdo[6-8]. Aunque aparecen nuevos métodos diagnósticos tales como la ecocardiografía y los péptidos natriuréticos $[9,10]$, la adecuada interpretación de los hallazgos clínicos sigue siendo fundamental para el diagnóstico.

Las preguntas frecuentes cuando se interroga a un paciente con insuficiencia cardiaca son[11]:

1. ¿Cuándo está quieto, se siente ahogado?

2. ¿Qué tanto puede caminar sin fatigarse?

3. ¿Se le hinchan los pies?

4. ¿Con cuántas almohadas duerme? 


\section{5. ¿Se despierta ahogado en la noche?}

La IC es un síndrome clínico que resulta de alteraciones estructurales y funcionales del llenado ventricular o eyección cardiaca. Las manifestaciones cardinales son disnea y fatiga, las cuales pueden limitar la tolerancia al ejercicio y la retención de fluidos que puede llevar a edema pulmonar, congestión esplácnica o edema periférico[3, 12].

Los pacientes con insuficiencia cardiaca pueden tener fracción de eyección reducida (ICFE-r) o fracción de eyección preservada (ICFE-p) $[3,12,13]$. Aunque menos frecuentes, los trastornos restrictivos pertenecen a esta última categoría. El diagnóstico diferencial de la IC debe hacerse con hipertensión pulmonar y pericarditis constrictiva.

ICFE-p es diagnosticada cuando síntomas y signos de insuficiencia cardiaca están presentes pero el ecocardiograma revela una fracción de eyección del ventrículo izquierdo (FEVI) mayor al 50\% en ausencia de una anormalidad valvular o pericárdica[14-16]. Está relacionada con la edad avanzada, sexo femenino, hipertensión arterial, diabetes, fibrilación auricular e hipertrofia ventricular izquierda, pero no con enfermedad arterial coronaria[16]. Más del 50\% de los pacientes con insuficiencia cardiaca tienen FEVI preservada[13, 17]. La mortalidad a 5 años entre pacientes con ICFE-r e ICFE-p es del 68\% y 65\% respectivamente[18]. Ni el examen físico ni la medición del BPN pueden distinguir la ICFE-r de la ICFE-p.[14]

Tabla 1. Sensibilidad y especificidad de los síntomas, signos y exámenes frecuentes asociados a IC.

\begin{tabular}{|l|c|c|}
\hline \multicolumn{1}{|c|}{ Signos y síntomas } & Sensibilidad (\%) & Especificidad (\%) \\
\hline Disnea con el ejercicio & $66-100$ & $17-83$ \\
\hline Disnea en reposo & 10 & 99 \\
\hline Ortopnea & $21-86$ & $74-99$ \\
\hline Disnea paroxística nocturna & $26-41$ & $76-99$ \\
\hline Fatigabilidad & 31 & 70 \\
\hline Edema & $23-55$ & $72-88$ \\
\hline FC $>100$ latidos/minuto en reposo & $5-36$ & $40-99$ \\
\hline Desplazamiento lateral del ápex & $27-92$ & $85-96$ \\
\hline Tercer Ruido (S3) & & \\
\hline FEVI $<50 \%$ & $3-52$ & $18-99$ \\
\hline FEVI $<30 \%$ & 78 & 88 \\
\hline Presiones elevadas de llenado & $30-95$ & $80-92$ \\
\hline Cuarto Ruido (S4) & & \\
\hline FEVI $<50 \%$ & $38-58$ & $48-97$ \\
\hline Presiones elevadas de llenado & $32-46$ & $45-85$ \\
\hline Estertores & $13-60$ & $77-96$ \\
\hline Hepatomegalia & $16-17$ & $96-97$ \\
\hline Presión venosa yugular elevada & $10-52$ & $70-97$ \\
\hline Test abdómino-yugular positivo & $24-26$ & $96-98$ \\
\hline BNP $\leq 100$ ng/L & 95 & 63 \\
\hline BNP $100-500$ ng/L & 85 & 86 \\
\hline NTproBNP $\leq 300$ ng/L & 99 & 43 \\
\hline NTproBNP 300-1800 ng/L & 90 & 76 \\
\hline
\end{tabular}

FC: Frecuencia Cardiaca, FEVI: Fracción de eyección del ventrículo izquierdo, BNP: Peptido natriurético cerebral tipo B,

El médico ha delegado el diagnóstico de la IC en la ecocardiografía y el Peptido natriurético cerebral tipo B (BNP), pero conocer que la búsqueda de galope S3 y la ingurgitación yugular brinda resultados de igual precisión para el diagnóstico, crea en el profesional la necesidad de agudizar sus destrezas. La sensibilidad y especificidad para cada síntoma, signo y examen útiles en el diagnóstico de la IC se revisa en la tabla $1[13,16,19-29]$.

Se ha determinado que no existe diferencia significativa entre la precisión del BNP y el fragmento $\mathrm{N}$-terminal del propéptido natriurético cerebral (NTproBNP) para el diagnóstico de IC[29]. Este último presenta una relación inversa con la función renal, lo que lleva a errores en el diagnóstico de IC cuando hay presencia de disfunción de este organo[30]. Otras causas de elevación de péptidos natriureticos incluyen cirrosis, hipertiroidismo, infecciones, isquemia cerebral, tromboembolismo pulmonar[31, 32], sexo femenino y envejecimiento[33]. Condiciones como la obesidad pueden producir niveles bajos de BNP[34].

De igual forma sucede con el ecocardiograma, el clínico descarga la responsabilidad del diagnóstico de la IC en este examen. Esto puede ser parcialmente útil. El ecocardiograma tiene excelente sensibilidad y especificidad para la detección de ICFE-r[35], pero más del 50\% de los casos de insuficiencia cardiaca son explicados por ICFE-p $[13,17]$ y los criterios diagnósticos de ésta aún no están estandarizados[15, 36-39].

Disnea es el síntoma cardinal de la IC. Se define como la percepción conciente de la respiración, molesta pero no dolorosa[20]. La disnea con el ejercicio aparece con frecuencia en etapas tempranas de la enfermedad, la cual progresa hasta producir disnea en reposo que se presenta en ausencia de actividad, estar acostado o dormido[20, 40]. Es predictor de múltiples hospitalizaciones y se relaciona de forma significativa $(\mathrm{p}<0.0001)$ con la mortalidad por IC[41]. Otras causas de disnea de origen no cardiaco incluyen enfermedades de las vías aéreas, de la pared torácica, de la vasculatura pulmonar, neuromusculares, alteraciones del parénquima pulmonar, anemia, enfermedad renal y enfermedades del pericardio[20, 42-45]. También puede presentarse disnea con hallazgos cardiovasculares y pulmonares normales en obesidad y ansiedad[40, 46].

Ortopnea se define como la disnea que ocurre en posición supina[47], se acompaña de tos nocturna y puede ser una manifestación tardía de la IC[48]. Se asocia a mayor número de hospitalizaciones[41]. Usualmente aparece posterior a la disnea de esfuerzo[20]. Otras causas de ortopnea son obesidad, ascitis, EPOC, asma, tumores mediastinales, apnea del sueño, parálisis diafragmática y embarazo[47, 49].

Disnea paroxística nocturna se refiere a un episodio de disnea y tos que puede estar acompañado o no de sibilancias que generalmente ocurre en la noche, despierta al paciente y lo obliga a sentarse. Aparece 1 a 3 horas luego de haberse acostado[47, 50]. Puede confundirse con asma nocturna o bronquitis crónica.

Fatiga es uno de los síntomas más frecuentes en pacientes con ICFE-r[51]. Se asocia con alteraciones del músculo esquelético por deficiencia en el flujo sanguíneo, en la función endotelial y en la estructura muscular[21]. Se considera un síntoma predictor de empeoramiento de la IC que tiene especial importancia ya que afecta la calidad de vida del paciente al limitar la actividad física[41, 48]. Otras causas de fatiga de origen no cardiaco son enfermedades hepáticas[52], anormalidades del sistema musculoesquelético, fibromialgia, miastenia gravis, esclerosis múltiple, hipotiroidismo, deficiencia de vitamina $\mathrm{D}$, anemia, hipercalcemia[53], obesidad, depresión, uso de betabloqueadores, estatinas, cáncer e infección por 
VIH[51, 54-56].

La IC es la causa más frecuente de edema bilateral y simétrico[13, 57, 58]. Al inicio compromete los tobillos, es blando, deja fóvea que desaparece en segundos y puede estar asociado a niveles bajos de albumina debido a la enteropatía perdedora de proteínas, disfunción hepática y renal asociadas $[59,60]$. Puede cursar con extremidades frías y comprometer la región sacra en pacientes acostados[47, 61, 62]. Otras causas de edema son enfermedad renal, cirrosis hepática, trastornos tiroideos, desnutrición, tumores pélvicos, trombosis de la vena cava inferior, insuficiencia venosa crónica, pericarditis constrictiva e hipertensión pulmonar[57, 63], medicamentos como calacioantagonistas, agonistas dopaminérgicos (Ropirinol, Pramipexol, bromocriptina) y anti inflamatorios no esteroideos[64].

Ingurgitación yugular, galope de S3, estertores pulmonares, reflujo hepatoyugular, ascitis y edema se relacionan con sobrecarga de volumen y presiones elevadas de llenado. Baja fracción de eyección se correlaciona con desplazamiento lateral del ápex, incremento en la presión de pulso, extremidades frías y taquicardia con pulso alternante[12].

El examen físico es el primer paso en la evaluación de la presencia y severidad de la retención de líquidos en pacientes con insuficiencia cardiaca, que se puede evaluar a través de la distensión venosa yugular, el test abdómino-yugular y la búsqueda de edema. Además de la congestión de órganos evidenciada por la presencia de estertores y hepatomegalia usualmente dolorosa[13, 16].

Con la introducción de nuevas tecnologías, el entrenamiento de muchos médicos ha llegado a ser menos eficiente en la interpretación de los signos y de los síntomas $[65,66]$. Al contrario de la percepción generalizada, las habilidades clínicas no mejoran con la edad, incluso puede ser más difícil su aprendizaje. Es por esto necesario repetir y reforzar las habilidades del estudiante en formación[67].

Solo el 50 a $60 \%$ de los clínicos son capaces de reconocer adecuadamente la ingurgitación yugular (IY), la presencia de está precede el inicio de los síntomas en la insuficiencia cardiaca. El hallazgo de ingurgitación yugular es el signo más fiable de sobre carga de volumen, el cual mejora su especificidad cuando coexiste con edema de miembros inferiores[47, 62, 68]. La presión elevada de las venas yugulares refleja el incremento en la presión de la aurícula derecha, la cual se correlaciona con elevada presión de llenado en el lado izquierdo del corazón y efectos adversos en los pacientes con insuficiencia cardiaca crónica[20,66].

La IY debe evaluarse con el paciente en posición supina recostado a $45^{\circ}$, se debe girar la cabeza a la izquierda para obtener mejor visualización de las venas derechas del cuello[69] y nunca debe evaluarse al lado izquierdo. El punto de referencia para realizar la medición es el ángulo esternal que se encuentra a $5 \mathrm{~cm}$ por arriba de la aurícula derecha y adyacente a la segunda costilla[70]. Se define como presión venosa yugular elevada si el punto más alto de la columna venosa es mayor a $3 \mathrm{~cm}$ con respecto a la paralela a nivel del ángulo esternal[71, 72].

Evaluar la IY en un paciente recostado puede representar dificultades para algunos clínicos, incluso puede haber variaciones de acuerdo a la amplitud anteroposterior del tórax entre paciente y paciente. Otro método más sencillo se basa en evaluar con el paciente sentado a $90^{\circ}$ y las piernas colgando, la presencia de las venas yugulares más arriba de la clavícula[73]. En un paciente de estatura promedio la aurícula derecha se encuentra a nivel del cuarto espacio intercostal y la distancia a la clavícula es de $12-16 \mathrm{~cm}$, una columna venosa yugular que aparezca por encima de la clavicula en el paciente sentado se considera IY[71, 72]. Otras causas de ingurgitación yugular diferentes a IC son taponamiento cardiaco, pericarditis constrictiva, insuficiencia cardiaca derecha, infarto del ventrículo derecho, valvulopatía tricúspidea, tumores mediastinicos[74] y síndrome de vena cava superior, pero todas estas patologías cursan sin disnea paroxística nocturna[75]. La IY puede ser difícil de observar en pacientes obesos o con cuello corto[76].

El test abdominoyugular se define como el aumento sostenido en la presión venosa yugular después de ejercer una presión de 20-35mmHg durante al menos 10 segundos en el cuadrante superior derecho con el paciente a $45^{\circ}$ [77]. Es un signo que refleja la presión atrial izquierda elevada y sobrecarga de volumen[20]. Es positivo cuando la yugular derecha se eleva más allá de $3 \mathrm{~cm}$ y se sostiene durante al menos 15 segundos luego de ser liberada la presión[78]. En un paciente normal, la presión venosa permanece sin cambio durante la maniobra o puede elevarse durante un latido cardiaco o dos[20, 62, 69, 71]. Los pacientes deben ser advertidos para abstenerse de sostener la respiración o hacer maniobras de valsalva. Otras causas de reflujo hepatoyugular son patologías que afecten la distensibilidad del ventrículo derecho (VD) como pericarditis constrictiva, cardiomiopatía restrictiva e infarto del VD[20].

El tercer ruido cardiaco (S3) proveniente del ventrículo izquierdo se oye mejor con la campana cerca del impulso apical, algunas veces solo es audible con el paciente en decúbito lateral izquierdo durante la espiración y aparece temprano en la diástole cercano al segundo ruido[20,50]. El S3 en pacientes menores de 40 años no se asocia a procesos patológicos. En pacientes mayores de 40 años indica una disfunción sistólica severa y está relacionado con sobrecarga de volumen[27]. La presencia de S3 se asocia a mayor número de hospitalizaciones y peor pronóstico[66]. Otras causas de S3 son insuficiencia mitral, defectos septales ventriculares, ductus arteriovenoso y embarazo[27].

El cuarto ruido (S4) aparece antes del primer ruido, se ausculta mejor con la campana en el ápex, borde esternal izquierdo o área subxifoidea e indica que el ventrículo izquierdo esta anormalmente rígido por hipertrofia o fibrosis. El hallazgo de S4 siempre revela anormalidad cardiaca y usualmente está presente en disfunción diastólica[28]. Otras causas de S4 son hipertensión arterial, infarto agudo de miocardio y enfermedad arterial coronaria crónica[28].

Los estertores deben auscultarse después de pedirle al paciente que tosa, en la insuficiencia cardiaca son inspiratorios, pueden ser gruesos o finos y se presentan en número de 4 a 9[79] en cada inspiración[50, 73]. Otras causas de estertores son neumonía, fibrosis intersticial, bronquitis crónica, asma y enfisema[80]. El $80 \%$ de los pacientes con IC tienen derrame pleural bilateral, el cual debe disminuir en las primeras 48 horas de haber iniciado el tratamiento con diuréticos $\mathrm{La}$ presencia de derrame pleural unilateral o el retardo en desaparecer después de la terapia son indicaciones de toracentesis[81].

Un latido apexiano por fuera de la línea medio clavicular con el paciente en posición supina incrementa la probabilidad de que el corazón está aumentado de tamaño en la radiografía de tórax y que la fracción de eyección está deprimida[20, 50,73].

Frecuencia cardiaca mayor de 100 latidos por minuto en reposo se asocia a disfunción regional y global del ventrículo izquierdo, se 
considera un factor de riesgo independiente para el desarrollo de IC y mortalidad por eventos cardiovasculares[82].

La presión de pulso (PP), se considera una medida de la rigidez arterial. Se define como la diferencia entre la presión sistólica y diastólica, es normal menor de $45 \mathrm{mmHg}[83]$. La PP tiende a incrementarse entre los 55 a 60 años cuando la presión arterial sistólica y la presión arterial diastólica tienden a aumentar y disminuir respectivamente[84]. Por cada $10 \mathrm{mmHg}$ que se eleve la presión del pulso aumenta en un 14\% el riesgo de desarrollar IC[85]. Valores bajos de PP se relacionan con una pobre FEVI[20] y mayor mortalidad en IC de origen isquémico y no isquémico[50]. Pulso alternante se refiere a la sucesión ininterrumpida de un latido fuerte y uno débil que es propio de la IC avanzada[86], se debe buscar con el tensiómetro insuflado a la presión sistólica que permite solo el paso de las pulsaciones fuertes quedando bloqueadas las pulsaciones débiles. Se evidencia a la palpación de la arteria radial con reducción del pulso a la mitad de la obtenida previamente cuando el tensiómetro esta insuflado. La presencia de pulso alternante sugiere una FEVI disminuida y un ventrículo izquierdo aumentado de tamaño[50, 71].

Otras manifestaciones tardías incluyen hígado congestionado y doloroso, edema intestinal y enteropatía perdedora de proteínas, que llevan a anorexia, nausea, saciedad temprana y de forma tardía a caquexia cardiaca[20], la cual se relaciona con una mortalidad del $50 \%$ a 18 meses[87]. La respiración de Cheyene Stokes está presente en el $40 \%$ de los pacientes con ICFE-r, se caracteriza por periodos de hipernea-apnea y es un factor de mal pronóstico, especialmente si se presenta durante el día[20,88]. Trastornos del sueño, confusión y desorientación, están relacionados con baja perfusión cerebral[89]. El hallazgo de cianosis labial sugiere excesiva actividad adrenérgica[89]. Ascitis e ictericia son manifestaciones tardías y se asocian con hígado congestionado o hipoxia hepática[90].

\section{Conclusión}

En las últimas dos décadas la destreza clínica de los médicos generales e inclusive de los internistas se ha deteriorado, solo unos pocos clínicos son capaces de reconocer los síntomas y signos clásicos de muchas enfermedades. Al contrario de la percepción generalizada estas destrezas no mejoran con la edad e incluso en este punto es más difícil desarrollarlas. Lo cual ha generado a una excesiva utilización de estudios paraclínicos llevando a costos elevados para el sistema de salud y perdiendo así la esencia medica de la relación médicopaciente. Por consiguiente es necesario establecer bases firmes de semiología en los médicos en formación.

\section{Financiamiento: ninguno}

Conflicto de intereses: los autores declaran no tener conflicto de intereses

\section{Referencias}

1. A. L. Bui, T. B. Horwich, and G. C. Fonarow, "Epidemiology and risk profile of heart failure," Nat Rev Cardiol, vol. 8, pp. 30-41, 01//print 2011.

2. A. S. Go, D. Mozaffarian, V. L. Roger, E. J. Benjamin, J. D. Berry, M. J. Blaha, et al., "Heart disease and stroke statistics--2014 update: a report from the American Heart Association," Circulation, vol. 129, p. e28, 2014.

3. C. W. Yancy, M. Jessup, B. Bozkurt, J. Butler, D. E. Casey, M. H. Drazner, et al., "2013 ACCF/AHA Guideline for the Management of Heart Failure: Executive SummaryA Report of the American College of Cardiology Foundation/American Heart Association
Task Force on Practice Guidelines," Journal of the American College of Cardiology, vol. 62, pp. 1495-1539, 2013.

4. E. A. Bocchi, "Heart Failure in South America," Current Cardiology Reviews, vol. 9, pp. 147-156, 05/

5. E. Hernández-Leiva, "Epidemiología del síndrome coronario agudo y la insuficiencia cardiaca en Latinoamérica," Revista Española de Cardiología, vol. 64, pp. 34-43, 2011.

6. V. Lazzarini, R. J. Mentz, M. Fiuzat, M. Metra, and C. M. O'connor, "Heart failure in elderly patients: distinctive features and unresolved issues," European journal of heart failure, vol. 15, pp. 717-723, 2013.

7. G. Y. Stein, A. Kremer, T. Shochat, T. Bental, R. Korenfeld, E. Abramson, et al., "The diversity of heart failure in a hospitalized population: the role of age," Journal of cardiac failure, vol. 18, pp. 645-653, 2012.

8. G. S. Bloomfield, F. A. Barasa, J. A. Doll, and E. J. Velazquez, "Heart failure in sub-Saharan Africa," Current cardiology reviews, vol. 9, pp. 157-173, 2013.

9. J. N. Kirkpatrick, M. A. Vannan, J. Narula, and R. M. Lang, "Echocardiography in heart failure: applications, utility, and new horizons," Journal of the American College of Cardiology, vol. 50, pp. 381-396, 2007.

10. L. B. Daniels and A. S. Maisel, "Natriuretic Peptides," Journal of the American College of Cardiology, vol. 50, pp. 2357-2368, //.

11. P. W. Rupert and O. Pippa, "Diagnosis and management of chronic heart failure," BMJ, vol. 348, 2014.

12. A. Heart Failure Society of, "Executive Summary: HFSA 2010 Comprehensive Heart Failure Practice Guideline," Journal of Cardiac Failure, vol. 16, pp. 475-539, 6// 2010.

13. A. Bruce, D. Robert, and A. Victoria, "Investigation and management of congestive heart failure," BMJ, vol. 341, 2010.

14. G. P. Aurigemma and W. H. Gaasch, "Diastolic Heart Failure," New England Journal of Medicine, vol. 351, pp. 1097-1105, 2004.

15. K. Hogg, K. Swedberg, and J. McMurray, "Heart failure with preserved left ventricular systolic function: epidemiology, clinical characteristics, and prognosis," Journal of the American College of Cardiology, vol. 43, pp. 317-327, 2/4/ 2004.

16. M. King, J. Kingery, and M. BARETTA CASEY, "Diagnosis and evaluation of heart failure," heart failure, vol. 100, p. 23, 2012.

17. F. P. Brouwers, R. A. de Boer, P. van der Harst, A. A. Voors, R. T. Gansevoort, S. J. Bakker, et al., "Incidence and epidemiology of new onset heart failure with preserved vs. reduced ejection fraction in a community-based cohort: 11-year follow-up of PREVEND," European heart journal, p. eht066, 2013.

18. T. E. Owan, D. O. Hodge, R. M. Herges, S. J. Jacobsen, V. L. Roger, and M. M. Redfield, "Trends in Prevalence and Outcome of Heart Failure with Preserved Ejection Fraction," New England Journal of Medicine, vol. 355, pp. 251-259, 2006.

19. J. Mant, J. Doust, A. Roalfe, P. Barton, M. Cowie, P. Glasziou, et al., "Systematic review and individual patient data meta-analysis of diagnosis of heart failure, with modelling of implications of different diagnostic strategies in primary care," 2009.

20. M. Gopal and B. Karnath, "Clinical diagnosis of heart failure," Hospital Physician, vol. 45, pp. 9-15, 2009.

21. R. Watson, C. Gibbs, and G. Lip, "ABC of heart failure: clinical features and complications," BMJ: British Medical Journal, vol. 320, p. 236, 2000.

22. C. Fonseca, H. Morais, T. Mota, F. Matias, C. Costa, A. GouveiaOliveira, et al., "The diagnosis of heart failure in primary care: value of symptoms and signs," European journal of heart failure, vol. 6, pp. 795-800, 2004.

23. A. Davie, C. Francis, L. Caruana, G. Sutherland, and J. McMurray, "Assessing diagnosis in heart failure: which features are any use?" 
Qjm, vol. 90, pp. 335-339, 1997.

24. A. M. Chang, A. S. Maisel, and J. E. Hollander, "Diagnosis of heart failure," Heart failure clinics, vol. 5, pp. 25-35, 2009.

25. G. Marcus, J. Vessey, M. V. Jordan, M. Huddleston, B. McKeown, I. L. Gerber, et al., "Relationship between accurate auscultation of a clinically useful third heart sound and level of experience," Archives of internal medicine, vol. 166, pp. 617-622, 2006.

26. R. Patel, D. Bushnell, and P. Sobotka, "Implications of an audible third heart sound in evaluating cardiac function," Western journal of medicine, vol. 158, p. 606, 1993.

27. N. J. Mehta and I. A. Khan, "Third heart sound: genesis and clinical importance," International Journal of Cardiology, vol. 97, pp. 183-186, 11// 2004.

28. S. Gupta and A. D. Michaels, "Relationship between accurate auscultation of the fourth heart sound and the level of physician experience," Clinical cardiology, vol. 32, pp. 69-75, 2009.

29. E. Roberts, A. J. Ludman, K. Dworzynski, A. Al-Mohammad, M. R. Cowie, J. J. V. McMurray, et al., The diagnostic accuracy of the natriuretic peptides in heart failure: systematic review and diagnostic meta-analysis in the acute care setting vol. 350, 2015.

30. S. Anwaruddin, D. M. Lloyd-Jones, A. Baggish, A. Chen, D. Krauser, R. Tung, et al., "Renal Function, Congestive Heart Failure, and Amino-Terminal Pro-Brain Natriuretic Peptide MeasurementResults From the ProBNP Investigation of Dyspnea in the Emergency Department (PRIDE) Study," Journal of the American College of Cardiology, vol. 47, pp. 91-97, 2006.

31. M. Burke and W. Cotts, "Interpretation of B-type natriuretic peptide in cardiac disease and other comorbid conditions," Heart Failure Reviews, vol. 12, pp. 23-36, 2007/03/01 2007.

32. S.-H. Tsai, Y.-Y. Lin, S.-J. Chu, C.-W. Hsu, and S.-M. Cheng, "Interpretation and Use of Natriuretic Peptides in NonCongestive Heart Failure Settings," Yonsei Medical Journal, vol. 51, pp. 151-163, 02/12

33. M. M. Redfield, R. J. Rodeheffer, S. J. Jacobsen, D. W. Mahoney, K. R. Bailey, and J. C. Burnett, "Plasma brain natriuretic peptide concentration: impact of age and gender," Journal of the American College of Cardiology, vol. 40, pp. 976-982, 2002.

34. S. R. Das, M. H. Drazner, D. L. Dries, G. L. Vega, H. G. Stanek, S. M. Abdullah, et al., "Impact of body mass and body composition on circulating levels of natriuretic peptides results from the Dallas Heart Study," Circulation, vol. 112, pp. 2163-2168, 2005.

35. D. E. Habash-Bseiso, R. Rokey, C. J. Berger, A. W. Weier, and P.-H. Chyou, "Accuracy of Noninvasive Ejection Fraction Measurement in a Large Community-Based Clinic," Clinical Medicine and Research, vol. 3, pp. 75-82, 11/02/received $02 / 10 /$ revised 03/09/accepted 2005.

36. P. Banerjee, A. L. Clark, and J. G. Cleland, "Diastolic heart failure: a difficult problem in the elderly," The American journal of geriatric cardiology, vol. 13, pp. 16-21, 2004.

37. M. R. Zile, W. H. Gaasch, J. D. Carroll, M. D. Feldman, G. P. Aurigemma, G. L. Schaer, et al., "Heart Failure With a Normal Ejection Fraction Is Measurement of Diastolic Function Necessary to Make the Diagnosis of Diastolic Heart Failure?," Circulation, vol. 104, pp. 779-782, 2001.

38. D. K. Gupta and S. D. Solomon, "Imaging in Heart Failure with Preserved Ejection Fraction,” Heart Failure Clinics, vol. 10, pp. 419-434, 7// 2014.

39. R. Wachter and F. Edelmann, "Diagnosis of Heart Failure with Preserved Ejection Fraction," Heart Failure Clinics, vol. 10, pp. 399-406, 7// 2014

40. A. Ahmed, R. M. Allman, W. S. Aronow, and J. F. DeLong,
"Diagnosis of heart failure in older adults: predictive value of dyspnea at rest," Archives of Gerontology and Geriatrics, vol. 38, pp. 297-307, 5// 2004.

41. I. Ekman, J. G. Cleland, K. Swedberg, A. Charlesworth, M. Metra, and P. A. Poole-Wilson, "Symptoms in patients with heart failure are prognostic predictors: insights from COMET," J Card Fail, vol. 11, pp. 288-92, May 2005.

42. N. Ambrosino, N. Carpene, and M. Gherardi, "Chronic respiratory care for neuromuscular diseases in adults," European respiratory journal, vol. 34, pp. 444-451, 2009.

43. E. Farrero, A. Antón, C. J. Egea, M. J. Almaraz, J. F. Masa, I. Utrabo, et al., "Guidelines for the Management of Respiratory Complications in Patients With Neuromuscular Disease," Archivos de Bronconeumología (English Edition), vol. 49, pp. 306-313, 7// 2013.

44. [44] M. A. Ko and G. E. Darling, "Acquired Paralysis of the Diaphragm," Thoracic Surgery Clinics, vol. 19, pp. 501-510, 11// 2009.

45. F. D. McCool and G. E. Tzelepis, "Dysfunction of the Diaphragm," New England Journal of Medicine, vol. 366, pp. 932-942, 2012.

46. M. B. Parshall, R. M. Schwartzstein, L. Adams, R. B. Banzett, H. L. Manning, J. Bourbeau, et al., "An official American Thoracic Society statement: update on the mechanisms, assessment, and management of dyspnea," American journal of respiratory and critical care medicine, vol. 185, pp. 435-452, 2012.

47. M. Gheorghiade, F. Follath, P. Ponikowski, J. H. Barsuk, J. E. Blair, J. G. Cleland, et al., "Assessing and grading congestion in acute heart failure: a scientific statement from the acute heart failure committee of the heart failure association of the European Society of Cardiology and endorsed by the European Society of Intensive Care Medicine," Eur J Heart Fail, vol. 12, pp. 423-33, May 2010.

48. A. C. Perez-Moreno, P. S. Jhund, M. R. Macdonald, M. C. Petrie, J. G. Cleland, M. Böhm, et al., "Fatigue as a predictor of outcome in patients with heart failure: analysis of CORONA (controlled rosuvastatin multinational trial in heart failure)," JACC: Heart Failure, vol. 2, pp. 187-197, 2014.

49. N. S. Yelgec, R. Atak, and S. Cay, "Severe Orthopnea is not Always Due to Heart Failure: A Case of Bilateral Diaphragm Paralysis," The Journal of Emergency Medicine, vol. 45, pp. 922-923, 12// 2013.

50. R. R. Baliga, G. W. Dec, and J. Narula, "Practice Guidelines for the Diagnosis and Management of Systolic Heart Failure in Lowand Middle-Income Countries," Global Heart, vol. 8, pp. 141$170,2013$.

51. A. M. Fink, S. L. Sullivan, J. J. Zerwic, and M. R. Piano, "Fatigue with systolic heart failure," J Cardiovasc Nurs, vol. 24, pp. 410-7, Sep-Oct 2009.

52. M. Hale, J. L. Newton, and D. E. J. Jones, "Fatigue in primary biliary cirrhosis," BMJ, vol. 345, 2012-10-22 11:58:07 2012.

53. B. K. Kinder and A. F. Stewart, "Hypercalcemia," Current Problems in Surgery, vol. 39, pp. 360-447, 4// 2002.

54. C. W. Yancy, M. Jessup, B. Bozkurt, J. Butler, D. E. Casey, Jr., M. H. Drazner, et al., "2013 ACCF/AHA guideline for the management of heart failure: a report of the American College of Cardiology Foundation/American Heart Association Task Force on Practice Guidelines," J Am Coll Cardiol, vol. 62, pp. e147-239, Oct 15 2013.

55. L. S. Evangelista, D. K. Moser, C. Westlake, N. Pike, A. TerGalstanyan, and K. Dracup, "Correlates of Fatigue in Patients With Heart Failure," Progress in Cardiovascular Nursing, vol. 23, pp. 12-17, 2008.

56. J. Barroso, J. Leserman, J. L. Harmon, B. Hammill, and B. W. Pence, 
"Fatigue in HIV-Infected People: A Three-Year Observational Study," Journal of Pain and Symptom Management.

57. H. W. Thaler, G. Wirnsberger, S. Pienaar, and R. E. Roller, "Bilateral leg edema in the elderly. Clinical considerations and treatment options," European Geriatric Medicine, vol. 1, pp. 353357, 12// 2010.

58. D. N. Papaliodis, M. A. Vanushkina, N. G. Richardson, and J. A. DiPreta, "The Foot and Ankle Examination," Medical Clinics of North America, vol. 98, pp. 181-204, 3// 2014.

59. J. L. Bonilla-Palomas, A. L. Gámez-López, M. Moreno-Conde, M. C. López-Ibáñez, M. Anguita-Sánchez, Á. G. de la Sacristana, et al., "Hypoalbuminemia in acute heart failure patients: causes and its impact on hospital and long-term mortality," Journal of cardiac failure, vol. 20, pp. 350-358, 2014.

60. R. Jabbour, H. Z. Ling, K. Norrington, N. Amaral, N. Zaman, S. Aggarwal, et al., "Serum albumin changes and multivariate dynamic risk modelling in chronic heart failure," International journal of cardiology, vol. 176, pp. 437-443, 2014.

61. D. C. Goff, Jr., D. M. Lloyd-Jones, G. Bennett, S. Coady, R. B. D’Agostino, Sr., R. Gibbons, et al., "2013 ACC/AHA Guideline on the Assessment of Cardiovascular Risk: A Report of the American College of Cardiology/American Heart Association Task Force on Practice Guidelines," Circulation, Nov 122013.

62. S. A. Hunt, W. T. Abraham, M. H. Chin, A. M. Feldman, G. S. Francis, T. G. Ganiats, et al., "2009 Focused update incorporated into the ACC/AHA 2005 Guidelines for the Diagnosis and Management of Heart Failure in Adults A Report of the American College of Cardiology Foundation/American Heart Association Task Force on Practice Guidelines Developed in Collaboration With the International Society for Heart and Lung Transplantation," J Am Coll Cardiol, vol. 53, pp. e1-e90, Apr 14 2009.

63. K. Muhyieddeen, W. Chen, and J. Varon, "A case of IVC filter thrombosis," Journal of Thrombosis and Thrombolysis, vol. 34, pp. 404-405, 2012/10/01 2012.

64. J. A. Zavala, R. P. Munhoz, and H. A. Teive, "Pramipexole-related chronic lower limb oedema in a patient with Parkinson's disease," J Clin Neurosci, vol. 19, pp. 1298-9, Sep 2012.

65. A. M. From, C. S. Lam, S. R. Pitta, P. V. Kumar, K. A. Balbissi, J. D. Booker, et al., "Bedside assessment of cardiac hemodynamics: the impact of noninvasive testing and examiner experience," Am J Med, vol. 124, pp. 1051-7, Nov 2011.

66. M. H. Drazner, J. E. Rame, L. W. Stevenson, and D. L. Dries, "Prognostic Importance of Elevated Jugular Venous Pressure and a Third Heart Sound in Patients with Heart Failure," New England Journal of Medicine, vol. 345, pp. 574-581, 2001.

67. D. Kasper, A. Fauci, S. Hauser, D. Longo, J. Jameson, and J. Loscalzo, Harrison's Principles of Internal Medicine 19/E (Vol.1 \& Vol.2): McGraw-Hill Education, 2015.

68. M. Patarroyo-Aponte and M. Colvin-Adams, "Evaluation of Patients with Heart Failure," Cardiology Clinics, vol. 32, pp. 4762, 2// 2014.

69. J. M. Liao, G. C. Stewart, R. F. Padera, A. L. Miller, and J. Loscalzo, "A Curious Case of Chest Pain," New England Journal of Medicine, vol. 369, pp. 1844-1850, 2013/11/07 2013.

70. M. M. APPLEFELD, "19 The Jugular Venous Pressure and Pulse Contour," Clinical methods: The history, physical, and laboratory examinations, p. 107, 1990.

71. C. V. Leier and K. Chatterjee, "The physical examination in heart failure-Part I," Congestive Heart Failure, vol. 13, pp. 41-47, 2007.

72. J. Sinisalo, J. Rapola, J. Rossinen, and M. Kupari, "Simplifying the estimation of jugular venous pressure," The American journal of cardiology, vol. 100, pp. 1779-1781, 2007.

73. C. V. Leier and K. Chatterjee, "The physical examination in heart failure-part II," Congestive Heart Failure, vol. 13, pp. 99-0103, 2007.

74. S. W. Corbett, L. B. Stack, and K. J. Knoop, "Chapter 7. Chest and Abdomen," in The Atlas of Emergency Medicine, 3e, K. J. Knoop, L. B. Stack, A. B. Storrow, and R. J. Thurman, Eds., ed New York, NY: The McGraw-Hill Companies, 2010.

75. J. M. S. C. Chiaco, N. I. Parikh, and D. J. Fergusson, "The jugular venous pressure revisited," Cleveland Clinic journal of medicine, vol. 80, p. 638, 2013.

76. T. Jang, C. Aubin, R. Naunheim, and D. Char, "Ultrasonography of the internal jugular vein in patients with dyspnea without jugular venous distention on physical examination," Annals of Emergency Medicine, vol. 44, pp. 160-168, 8// 2004.

77. J. Wiese, "The abdominojugular reflux sign," The American Journal of Medicine, vol. 109, pp. 59-61, 7/28/ 2000.

78. G. A. Ewy, "The abdominojugular test: technique and hemodynamic correlates," Annals of internal medicine, vol. 109, pp. 456-460, 1988.

79. A. Bohadana, G. Izbicki, and S. S. Kraman, "Fundamentals of Lung Auscultation," New England Journal of Medicine, vol. 370, pp. 744-751, 2014.

80. P. Piirila and A. Sovijarvi, "Crackles: recording, analysis and clinical significance," European Respiratory Journal, vol. 8, pp. 2139-2148, 1995.

81. R. W. Light, "Pleural Effusion," New England Journal of Medicine, vol. 346, pp. 1971-1977, 2002/06/20 2002.

82. A. Opdahl, B. Ambale Venkatesh, V. R. Fernandes, C. O. Wu, K. Nasir, E. Y. Choi, et al., "Resting Heart Rate as Predictor for Left Ventricular Dysfunction and Heart Failure: MESA (MultiEthnic Study of Atherosclerosis)," J Am Coll Cardiol, vol. 63, pp. 1182-9, Apr 12014.

83. C. U. Chae, M. A. Pfeffer, R. J. Glynn, G. F. Mitchell, J. O. Taylor, and C. H. Hennekens, "Increased pulse pressure and risk of heart failure in the elderly," Jama, vol. 281, pp. 634-643, 1999.

84. W. J. Mosley, 2nd, P. Greenland, D. B. Garside, and D. M. LloydJones, "Predictive utility of pulse pressure and other blood pressure measures for cardiovascular outcomes," Hypertension, vol. 49, pp. 1256-64, Jun 2007.

85. V. Vaccarino, T. R. Holford, and H. M. Krumholz, "Pulse pressure and risk for myocardial infarction and heart failure in the elderly," Journal of the American College of Cardiology, vol. 36, pp. 130-138, 2000.

86. P. Szymański, M. Lipczyńska, A. Klisiewicz, and P. Hoffman, “"Like a sound and its echo'"' Heart, vol. 100, p. 83, January 1, 20142014.

87. M. Valentova, S. von Haehling, C. Krause, N. Ebner, L. Steinbeck, L. Cramer, et al., "Cardiac cachexia is associated with right ventricular failure and liver dysfunction," Int J Cardiol, vol. 169, pp. 219-24, Nov 52013.

88. A. J. Quaranta, G. E. D’Alonzo, and S. L. Krachman, "CHeynestokes respiration during sleep in congestive heart failure," Chest, vol. 111, pp. 467-473, 1997.

89. K. Dickstein, M. Authors/Task Force, A. Cohen-Solal, G. Filippatos, J. J. V. McMurray, P. Ponikowski, et al., "ESC Guidelines for the diagnosis and treatment of acute and chronic heart failure 2008 ”, European Journal of Heart Failure, vol. 10, pp. 933-989, 2008.

90. R. van Lingen, U. Warshow, H. R. Dalton, and S. H. Hussaini, "Jaundice as a presentation of heart failure," Journal of the Royal Society of Medicine, vol. 98, pp. 357-359, 2005. 